ONDERZOEKSRAPPORT NR 9421

\title{
Extending the Shifting Bottleneck \\ Procedure to Real-Life Applications
}

by

Philip IVENS

Marc LAMBRECHT

$\mathrm{D} / 1994 / 2376 / 23$ 


\title{
Extending the Shifting Bottleneck Procedure to Real-Life Applications ${ }^{*}$
}

\author{
Philip Ivens \\ Marc Lambrecht \\ Department of Applied Economic Sciences \\ K.U.Leuven, Belgium
}

\begin{abstract}
Much research has been devoted to the job shop scheduling problem since its introduction in the late 50's. Despite these efforts, even moderate sized benchmarking problems remained unsolved for many years. Given the complexity of the job shop scheduling problem, there is little hope for solving large real-life problems optimally within reasonable time. We therefore rely on heuristics, of which the Shifting Bottleneck Procedure, developed by Adams et al. (1988), is performing excellently. We examine several extensions of the Shifting Bottleneck Procedure towards real-life applications. We introduce due dates, release dates, assembly structures, split structures, overlapping operations, setup times, transportation times, parallel machines and beginning inventory. This generalized shifting bottleneck procedure is compared with priority dispatching rules on a set of large test problems.
\end{abstract}

\section{Introduction}

Most production planning and control systems focus on managing the material flow. Manufacturing Resources Planning (MRP) is the most widespread example of such a planning system. A major weakness of MRP is the way in which capacity issues are dealt with. At the same time it is obvious that the effective use of plant capacity is an important determinant for manufacturing success. Measures of shop performance such as due date performance, flow time reduction and reduced inventory levels are closely linked to capacity issues. This observation initiated a renewed interest in capacity oriented planning systems or more specifically finite schedulers. This approach focuses on production processes, specifying for each operation when it has to be performed and by which resource, explicitly taking into account the limited availability of the various resources.

\footnotetext{
* This research is supported by the Belgian National Science Foundation under contract NFWO (FKFO) 2.0053 .93
} 
This automatically promotes the job shop scheduling problem as the theoretical starting point. The job shop scheduling problem has been subject of numerous research efforts. Both optimal and heuristic solution procedures are proposed in literature. Recent integer programming based models include Balas (1985) and Applegate \& Cook (1991). Lageweg et al. (1977), Barker \& McMahon (1985), Carlier \& Pinson (1989), Brucker et al. (1991), Brucker et al. (1992) propose implicit enumeration methods for solving the job shop problem. Despite numerous efforts, only moderate sized problems can be solved optimally. One of the most common heuristic solution procedures is priority dispatching. This approach generates active or non-delay schedules based on well known priority dispatching rules such as SPT (shortest processing time), MWR (most work remaining), FCFS (first come, first served), to mention only a few. Simulated annealing is an iterative improvement scheme that also has been applied to the job shop scheduling problem (Van Laarhoven et al. (1992)).

Adams, Balas \& Zawack (1988) introduced the very promising Shifting Bottleneck Procedure (SBP) based on the disjunctive graph formulation. Because of the SBP's good balance between computational complexity and the quality of the generated schedules, it was decided to use this procedure as the core of the algorithm presented in this paper.

The purpose of this paper is to extend the disjunctive graph representation and the Shifting Bottleneck Procedure so that non-standard job shop scheduling problems can be solved. Indeed, real-life applications are characterized by features such as : due dates, release dates, assembly structures, split structures, overlapping operations, setup times, transit times, parallel machines and beginning inventories.

This paper is organized as follows. In section 2 the general scheduling problem and the transformation to its corresponding extended disjunctive graph will be described . In section 3 we propose a Shifting Bottleneck Procedure to solve the extended disjunctive graph. In section 4 , we report on the computational experience with the algorithm. Section 5 concludes the paper.

\section{Transforming Practical Problems into an Extended Disjunctive Graph.}

\subsection{The Extended Disjunctive Graph}

An activity-on-the-node disjunctive graph $\mathrm{DG}=(\mathrm{N}, \mathrm{A}, \mathrm{E})$ for the standard job shop scheduling problem is defined as follows. $N=N^{\prime} \cup\{b\} \cup\{e\}$ with $N^{\prime}$ the set of nodes each representing one operation, and $\{\mathrm{b}\}$ and $\{\mathrm{e}\}$ are dummy nodes indicating the start and end of the schedule. Each node $i \in N^{\prime}$ has a label $p_{i}$, the processing time of operation $i$. A is the set of directed (conjunctive) arcs, representing precedence relations between nodes $(i, j)$ of $N$. E is the set of undirected (disjunctive) arcs, which represent precedence relationships between nodes $(i, j)$ of $N^{\prime}$ that require the same resource. Initially, the arcs $E$ are undirected. A solution to the problem corresponds with choosing a direction for each arc in $E$. Let $E^{\prime}$ be such a selection, i.e. a set of directed arcs. $D G^{\prime}=\left(N, A, E^{\prime}\right)$ is the graph obtained when replacing the disjunctive arcs in $G$ by the selection $E^{\prime}$. The longest path in $D^{\prime}$ from $\{b\}$ to $\{e\}$ corresponds to the makespan of the schedule. A feasible solution requires that $D G^{\prime}$ is acyclic. The set $E\left(E^{\prime}\right)$ can be partioned into $m$ subsets $E_{1}, \ldots, E_{m}\left(E_{1}{ }^{\prime}, \ldots, E_{m}{ }^{\prime}\right)$, each set $E_{k}$ represents the arcs connecting 
nodes which require the same resource.

To model non-standard job shop problems, we define the extended disjunctive graph $\mathrm{EDG}=(\mathrm{N}, \mathrm{A}, \mathrm{E})$. The main difference between the DG and the EDG is that we allow parallel machining and that all arcs in $\mathrm{A}$ and $\mathrm{E}$ receive a label to represent finish-start precedence relationships. The finish-start precedence relationships are required to model e.g. transportations times (or other delays), sequence dependent setup times and overlapping. Each $\operatorname{arc}(\mathrm{i}, \mathrm{j}) \in A$ has a label $F S_{\mathrm{ij}}$ to model the finish-start relation (e.g. to model delays or overlapping). $F S_{i j}$ is unrestricted in sign. Similarly, each arc $(i, j) \in E$ has a label $S_{i j}$ to model sequence dependent setup times. A detailed overview of the use of these labels is presented below (see section 2.2.). Since parallel machining is allowed, not all arcs of $E$ have to be selected in a solution. Let $k$ represent a resource, $k=\{1, \ldots, K\}$. Suppose further that $R_{k}$ units are available of a specific resource $k$. Each node $i$ requiring resource $k$, denoted by $k(i)$, has to be assigned to one of the $R_{k(i)}$ units available. We denote such an assignment of node $i$ by $X_{i z}=1$ if $i$ is assigned to the $z^{\text {th }}$ unit of resource $k(i), X_{i z}=0$ else. Then, a solution $E^{\prime}$ consists only of arcs (i,j) such that $X_{\mathrm{iz}}=\mathrm{X}_{\mathrm{j}}=1$, other arcs are discarded. This means that only selections of disjunctive arcs between nodes assigned to the same unit of resource $k$ are valid. The solution corresponding to a valid selection can be represented by the acyclic graph $E D G^{\prime}=\left(N, A, E^{\prime}\right)$. The makespan equals the longest path in $E D G^{\prime}$ from $\{b\}$ to $\{e\}$, taking into account the labels $F_{\mathrm{ij}}$ and $S_{\mathrm{ij}}$ of the arcs. The details of this longest path computation are given in section 3 .

\subsection{Practical Production Scheduling Problems}

In this section, we give a brief description of general production scheduling problems encountered in practice. We indicate how these problems can be transformed to an extended disjunctive graph. We will illustrate this transformation by a small example.

The production scheduling problem addressed here is one of scheduling orders on a set of resources. An order is a batch of identical products. We assume that the size of the batch is given. In other words, the planner predefines the size of the manufacturing order. Each product consists of a number of components, represented by a Bill of Material (BOM). In figure 1 we represent our example problem consisting of two products.
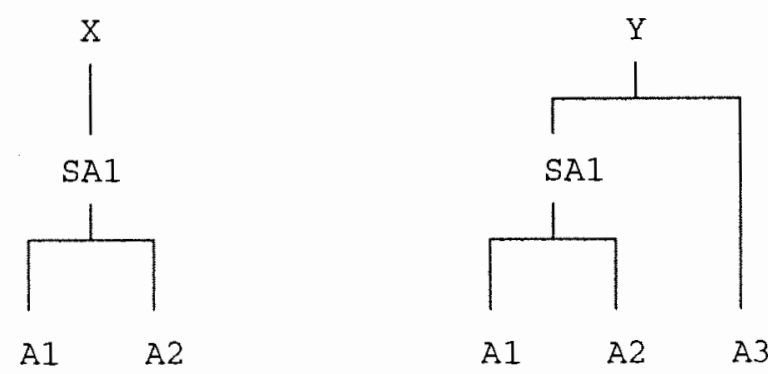

Figure 1: The BOM of our Example Problem 
Part SA1 is a subassembly consisting of parts $A 1$ and $A 2$. Product $X$ requires one unit of SA1. Product $\mathrm{Y}$ is an assembly of SA1 and part A3. Each part is characterized by a routing, indicating the sequence of processes. Each process is performed on a specific resource (a resource may be a machine, a worker of a tool). The information is summarized in table $\mathrm{I}$. Suppose that we have 6 resources M1 to M6. Two units of M1 and M3 are available and for all other resources there is only one unit available.

$\begin{array}{llll}\text { part } & \text { process } & \text { resource } & \text { time/unit } \\ \text { A1 } & \text { P1 } & \text { M1 } & 8 \\ & \text { P2 } & \text { M2 } & 11 \\ & \text { P3 } & \text { M3 } & 23 \\ \text { A2 } & \text { P4 } & \text { M1 } & 7 \\ & \text { P5 } & \text { M3 } & 9 \\ & \text { P6 } & \text { M4 } & 19 \\ \text { SA1 } & \text { P7 } & \text { M5 } & 17 \\ \text { X } & \text { P8 } & \text { M6 } & 6 \\ \text { A3 } & \text { P9 } & \text { M1 } & 3 \\ & \text { P10 } & \text { M3 } & 7 \\ \text { Y } & \text { P11 } & \text { M5 } & 12 \\ & \text { P12 } & \text { M6 } & 12\end{array}$

Table I : Routing Data of our Example Problem

We assume that each process needs only one resource. The production time per unit on the resource is given. For example, part A1 requires 3 processes. The first process, P1, is performed on machine $\mathrm{M} 1$ and requires 8 time units per unit produced. Next there is process $\mathrm{P} 2$ on resource $\mathrm{M} 2$ for 11 time units and finally, process P3 requires machine M3 for 23 time units.

Suppose that we have orders for products $X$ and $Y$. The BOM and the list of processes can be merged into a Bill of Processes (BOP). This is illustrated on figure 2.

Each block represents a unique part/process combination. In the upper left corner of a block, the process is given. In the lower left corner, the required resource is indicated and the number on the right side is the processing time per unit. The BOP will be the basis for the transformation of a scheduling problem into an extended disjunctive graph. 

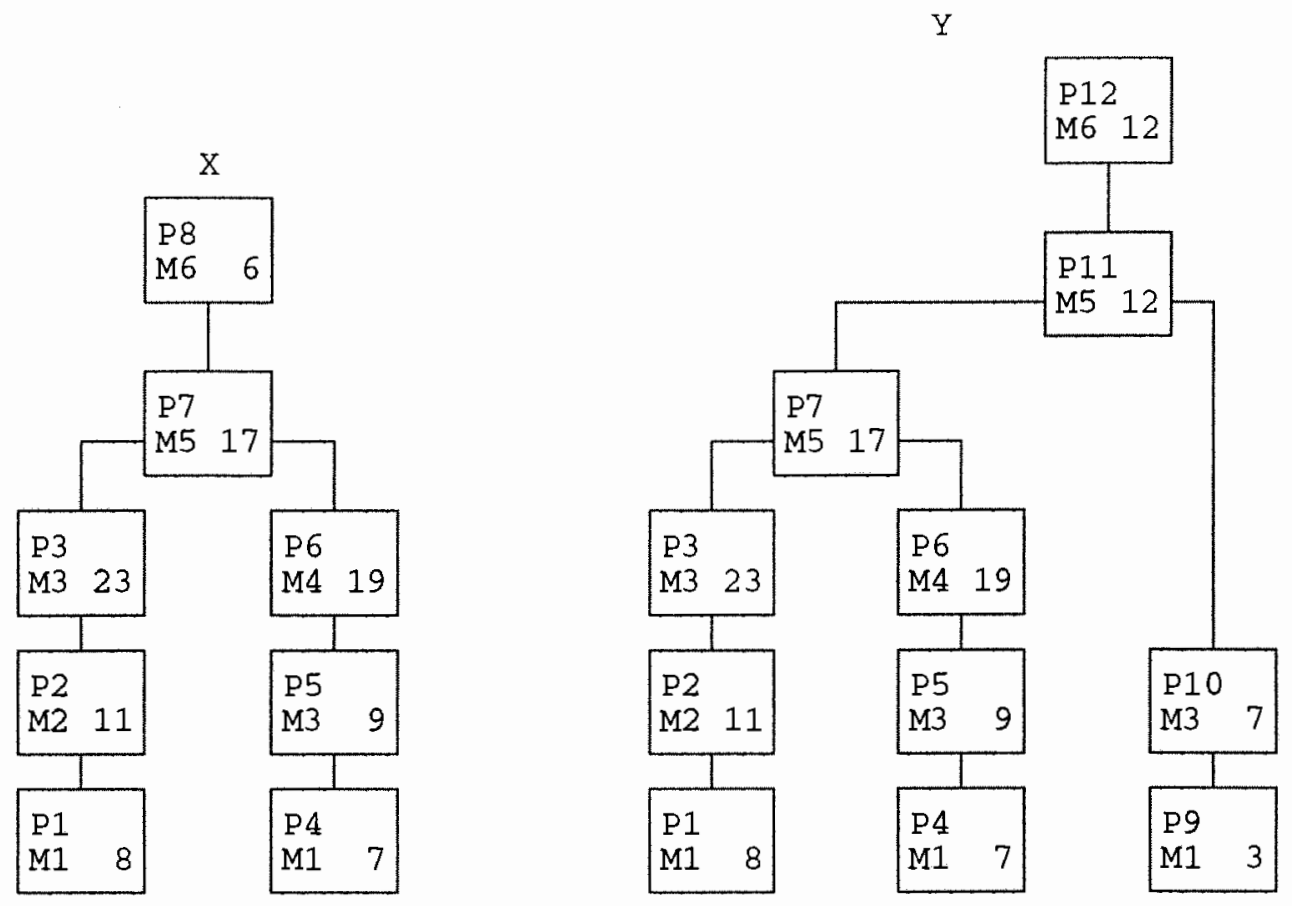

Figure 2 : The Bill of Processes

Suppose now that we have 3 orders in a certain planning period :

\begin{tabular}{|c|c|c|}
\hline Order & Product & Quantity \\
\hline O1 & $\mathrm{X}$ & 10 \\
\hline $\mathrm{O} 2$ & $\mathrm{Y}$ & 10 \\
\hline $\mathrm{O} 3$ & $\mathrm{X}$ & 20 \\
\hline
\end{tabular}

Table II : Order Data for our example problem

The resulting extended disjunctive graph is shown in figure 3. Each node in figure corresponds to a block of the BOP. The weight of a node is the processing time of the process multiplied by the size of the order. For example, nodes 1 to 8 correspond to order O1. Node 1 corresponds to process Pl and has a weight of $8 * 10=80$. For the time being we do not label the arcs, this will be relaxed later on. Each node represents one process and we assume that the processing takes place without preemption. If a process has started on a particular order, this process cannot be interrupted until all units are completed. The encircled nodes represent processes that require the same resource. The nodes in these subsets are linked through the undirected (disjunctive) arcs. For reasons of clarity, these arcs are not shown on figure 3 . 


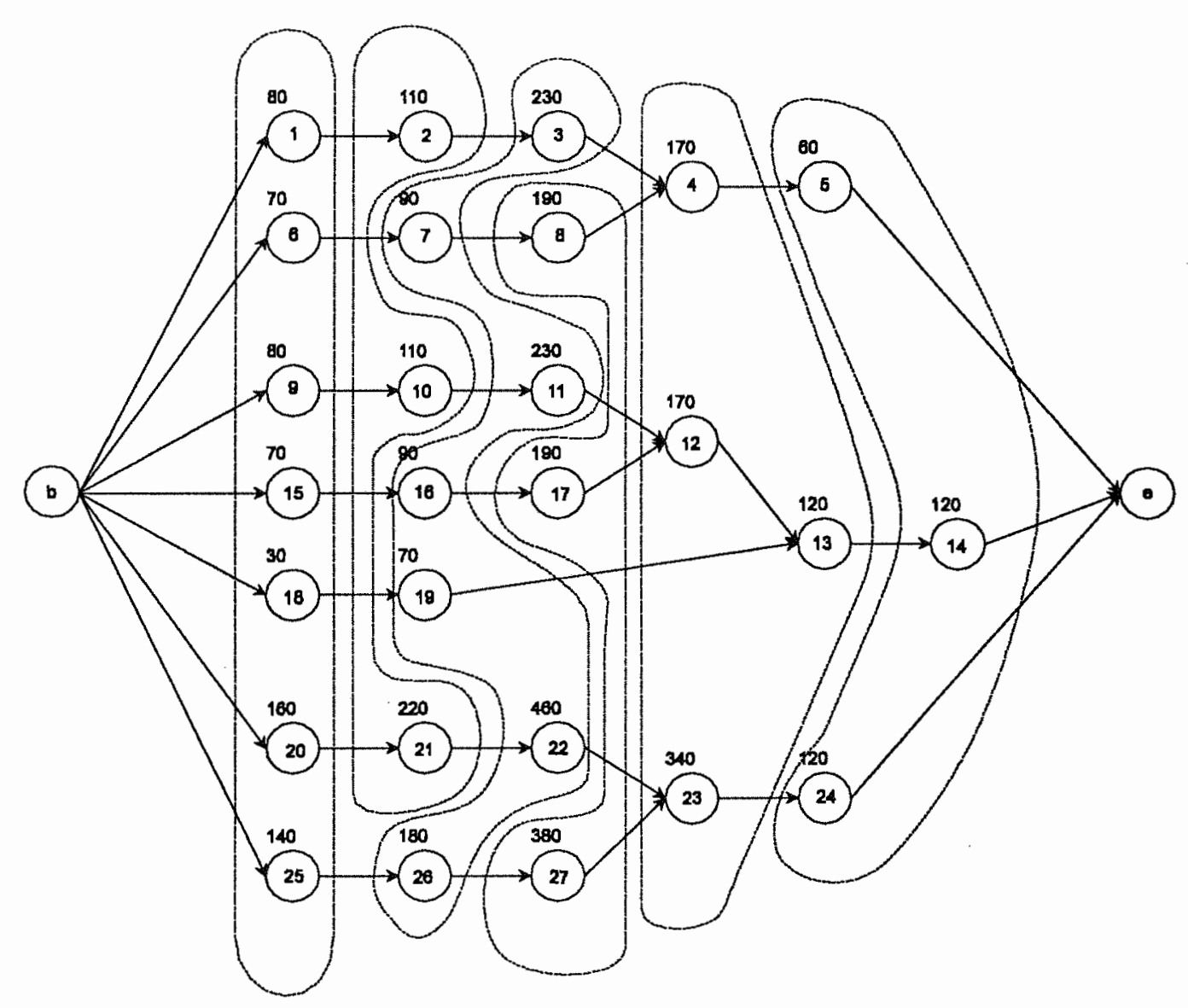

Figure 3 : The Extended Disjunctive Graph

\subsection{Further Modelling Topics}

A real-life problem, however, is more complicated than our example problem. In this section, it is shown how practical features such as assemblies, splits, overlapping processes, setup times, release dates and due dates can be modelled and represented by means of the extended disjunctive graph. Our example problem will be used to illustrate these extensions.

\subsubsection{Assembly and Split Structures}

Product assemblies and splits can easily be modelled by allowing multiple predecessors or successors. If certain parts require several components of the same type then we simply have to adjust the batch size of the corresponding component.

\subsubsection{Overiapping Processes}

It is common practice to allow overlapping, i.e., units do not have to wait till the whole process batch is finished, instead, products flow from one machine to the other in several smaller transfer batches. We therefore define a transfer batch of size $\mathrm{TB}_{\mathrm{ij}}$. As soon as $\mathrm{TB}_{\mathrm{ij}}$ 
units are processed at a resource required by $\mathrm{i}$, the units can move to the next process $\mathrm{j}$. An overlap is a negative finish-start relation, $\mathrm{FS}_{\mathrm{ij}}$, between successive operations in the EDG network.

Let $\mathrm{i}$ and $\mathrm{j}$ be two successive processes. We further define $\mathrm{pu}_{\mathrm{i}}$ and $\mathrm{pu}_{\mathrm{j}}$ as the processing times per unit of processes $i$ and $j$. The process batch size is $B$. If $B$ is not divisible by $T_{i j}$, there will be less than $\mathrm{TB}_{\mathrm{ij}}$ units in the last transport.

Let $\begin{aligned} \mathrm{TB}_{\mathrm{ij}}{ }^{\prime} & =\mathrm{TB}_{\mathrm{ij}} & & \text { if } \mathrm{B} \bmod \mathrm{TB}_{\mathrm{ij}}=0, \\ & =\mathrm{B} \bmod \mathrm{TB}_{\mathrm{ij}} & & \text { otherwise }\end{aligned}$

Note that we do not allow preemption of processing. We now derive the FS-relationship for 3 cases : $\mathrm{pu}_{\mathrm{i}}=\mathrm{pu}_{\mathrm{j}}, \mathrm{pu}_{\mathrm{i}}<\mathrm{pu}_{\mathrm{j}}, \mathrm{pu}_{\mathrm{i}}>\mathrm{pu}_{\mathrm{j}}$.

$\underline{\mathrm{pu}}_{\mathrm{i}}=\mathrm{pu}_{\mathrm{j}}$

In this case, $\mathrm{FS}_{\mathrm{ij}}=\left(\mathrm{TB}_{\mathrm{ij}}-\mathrm{B}\right) \mathrm{pu}_{\mathrm{i}}$, as shown in figure 4. Each block represents one transfer batch. Process $\mathrm{j}$ can start after processing $\mathrm{TB}_{\mathrm{ij}}$ units of the batch at the previous resource. The entire processing on the second resource can take place without preemption : when the processing of a transfer batch on the second resource is finished, there will be a new incoming transfer batch available from the first resource because both processes have the same speed.

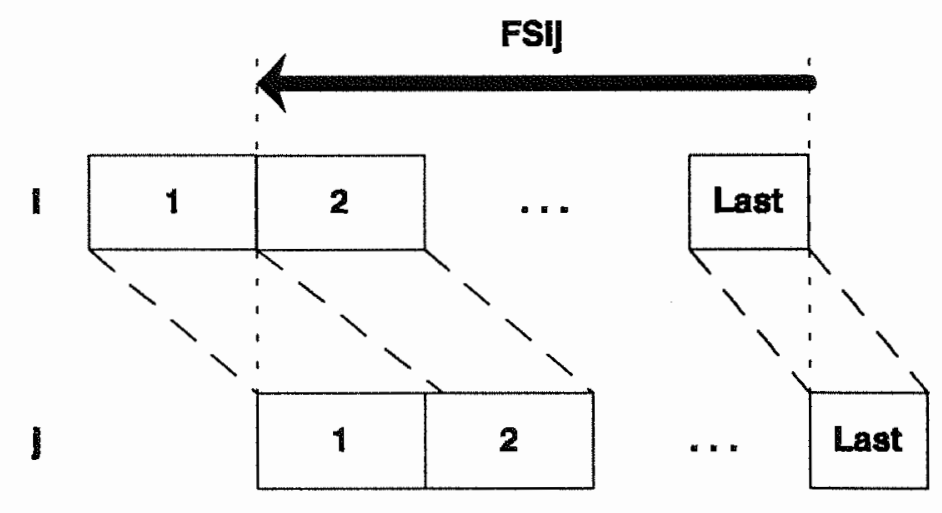

Figure 4 : Overlapping for $p u_{i}=p u_{j}$

$\underline{\mathrm{pu}}_{\mathrm{i}}<\mathrm{pu}_{\mathrm{j}}$

Again, $\mathrm{FS}_{\mathrm{ij}}=\left(\mathrm{TB}_{\mathrm{ij}} \mathrm{B}\right) \mathrm{pu}_{\mathrm{i}}$, see figure 5. Once started, the second resource will never wait for parts coming from the first resource, since the production speed of $i$ is higher than the speed of $\mathrm{j}$. 


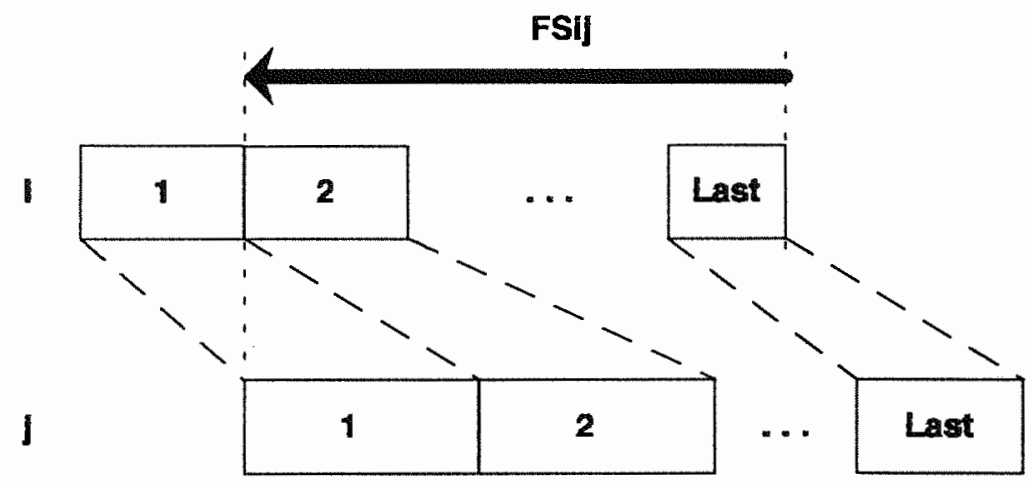

Figure 5 : Overlapping for $p u_{i}<p u_{j}$

$\underline{p u}_{i}>\mathrm{pu}_{\mathrm{j}}$

In this case, the second process cannot start $\mathrm{TB}_{\mathrm{ij}} \mathrm{pu}_{\mathrm{i}}$ time units after the start of the first process, because the second resource would have to wait for incoming units due to the fact that the production speed of process $i$ is lower than that of process $j$. It is required that the processing of the last transfer batch on the second resource does not occur earlier than the finish of the entire batch on the first resource, see figure 6 , or, $\mathrm{FS}_{\mathrm{ij}}=\left(\mathrm{TB}_{\mathrm{ij}}{ }^{\mathrm{i}}-\mathrm{B}\right) \mathrm{pu}_{\mathrm{j}}$.

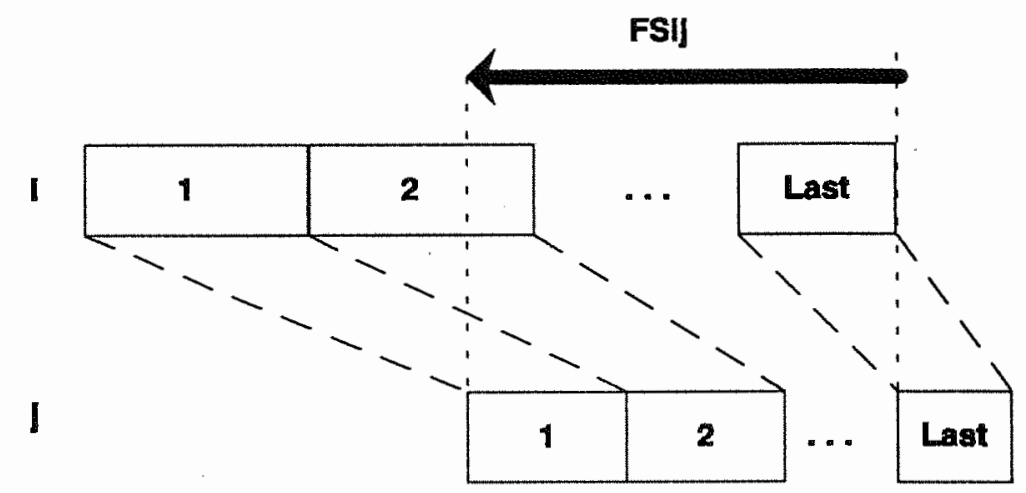

Figure 6 : Overlapping for $p u_{i}>p u_{j}$

The following formula includes all 3 cases :

$\mathrm{FS}_{\mathrm{ij}}=\max \left(\left(\mathrm{TB}_{\mathrm{ij}}-\mathrm{B}\right) \mathrm{pu}_{\mathrm{i}},\left(\mathrm{TB}_{\mathrm{ij}}{ }^{\prime}-\mathrm{B}\right) \mathrm{pu}_{\mathrm{j}}\right)$

\subsubsection{Transit Time}

Up till now, we have assumed that it takes no time to move a transfer batch from one resource to another. Suppose $\mathrm{T}_{\mathrm{ij}}$ time units are required for internal transportation or any other type of delay, the finish-start relations can be modified as follows : 
$\mathrm{FS}_{\mathrm{ij}}=\max \left(\left(\mathrm{TB}_{\mathrm{ij}}-\mathrm{B}\right) \mathrm{pu}_{\mathrm{i}},\left(\mathrm{TB}_{\mathrm{ij}}{ }^{\prime}-\mathrm{B}\right) \mathrm{pu}_{\mathrm{j}}\right)+\mathrm{T}_{\mathrm{ij}}$

\subsubsection{Release Dates and Due Dates}

Assume now that an order $\mathrm{i}$ has a release date $\mathrm{REL}_{\mathrm{i}}$ and a due date $\mathrm{DUE}_{\mathrm{i}}$. The order cannot be started before $R L_{i}$ and needs to be finished ideally before $D E_{i}$. We will show that, the problem of minimizing the maximum lateness is easily modelled by the extended disjunctive graph by assigning labels to the arcs connecting the dummy nodes with the other nodes.

A release date of an order is a finish-start relation between the starting point of the schedule (node $\{b\}$ ) and its first process(es). Let $F_{i}$ the set of all first processes of order $i$. Then, each $\operatorname{arc}(\{b\}, j)$ for all $j \in F_{i}$ will receive a weight $F S_{\{b\} j}=R E L_{i j}$.

Let $\mathrm{L}_{\mathrm{i}}$ be the set of all final processes of order $\mathrm{i}$. To model a minimum maximum lateness problem by the extended disjunctive graph, we weight all arcs leading to the dummy finish node. For each order $\mathrm{i}, \mathrm{FS}_{\mathrm{j}\{\mathrm{e}\}}=\mathrm{Z}$-DUE $\forall \mathrm{j} \in \mathrm{L}_{\mathrm{i}}$, with $\mathrm{Z}$ some constant. Let $\mathrm{C}_{\mathrm{i}}$ be the maximum of all completion times of the last processes of order i. By adding the final FSrelation to $C_{i} \forall$ orders $i$, the length of the schedule will be

$$
\operatorname{Max}_{i} C_{i}+Z-D_{i}=\operatorname{Max}_{i} C_{i}-D E_{i}
$$

which is exactly the min Lmax objective.

\subsubsection{Processing and Setup Related Time}

In our model, it is possible that processes have a setup time. We make a distinction between sequence independent and sequence dependent setup times. Sequence independent setup times are simply added to the processing time. Sequence dependent setup times are modeled through the finish start relations represented by the arcs in the set $\mathrm{E}$. Whenever there is a sequence dependent setup time caused by a changeover from process $i$ to process $j$, the arc $(\mathrm{i}, \mathrm{j})$ in $\mathrm{E}$ will receive a label equal to that setup time.

\subsubsection{Initial Inventory}

Practical problems are not isolated in time. Most large scale scheduling systems divide the time into smaller scheduling periods (e.g. one week). Because of the continuity of the production, it is possible that some orders are not finished at the end of a period. In that case, there will be beginning inventory in the next period : some orders will be in process or waiting for processing, and they have to be incorporated in the planning. This is achieved by generating only those parts of the network that still need to be processed.

\subsubsection{A Numerical Example}

We will now further complicate the example of figure 3 by introducing release dates, due dates, overlapping and setup times. This will result in an EDG with non-zero FS-precedence relationships. Suppose that the orders have the following release dates and due dates. 


\begin{tabular}{|c|c|c|}
\hline Order & Release Date & Due Date \\
\hline O1 & 0 & 900 \\
\hline O2 & 200 & 1200 \\
\hline O3 & 300 & 2000 \\
\hline
\end{tabular}

Table III : The Release Dates and Due Dates of our Example Problem

For simplicity, we assume transfer batches of size 1 for each process. The setup times of resources M2 and M3 are respectively 30 and 100. Other resources have no setup time. Setup times are sequence independent. These assumptions lead to the EDG shown on figure 7.

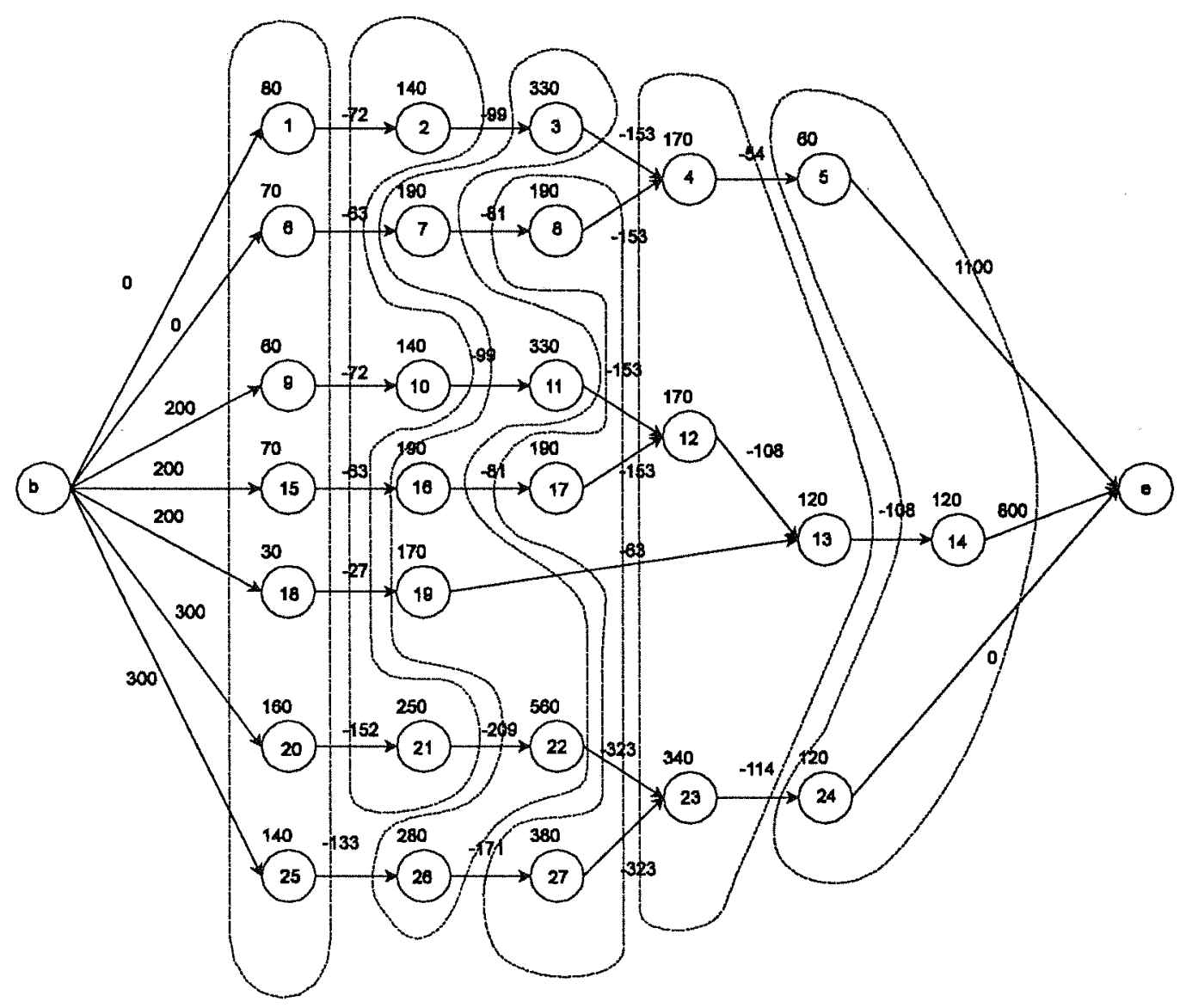

Figure 7 : The Extended Disjunctive Graph with Non-Zero FS-relationships

We will take $\mathrm{O} 2$ (order 2) as an example. The arcs connecting the dummy start node and the first processes of order $\mathrm{O} 2$ receive a weight of 200 to model the release date of the order (arcs b-9, b-15 and b-18). This will prevent the order from starting before time 200. To model the due date, we take some constant, e.g. the maximum of all due dates which is 2000 , 
and subtract the due date of $\mathrm{O} 2,1200$, from this number. The arc connecting the last process of $\mathrm{O} 2$ with the dummy end node (14-e) receives the weight $2000-1200=800$. The arc linking node 9 and 10 has a weight of -72 to model the overlap between these two processes. From formula (9) we obtain $\mathrm{FS}_{9,10}=\max ((1-10) * 8,(1-10) * 11)=-72$. Since resource M2 requires a setup time of 30 time units, the weight of node 10 is 140 , consisting of 30 time units of setup time and $10^{*} 11$ time units of processing time.

\section{Solving the Extended Disjunctive Graph with the Shifting Bottleneck Procedure}

In this section, we describe an adapted version of the shifting bottleneck procedure to solve problems represented by the EDG. First, we give a brief overview of the SBP for the standard job shop problem.

\subsection{The SBP for $D G$}

In the standard job shop problem, only one unit is available of each resource, and the features described in section 2.3. are absent. The shifting bottleneck procedure for this problem can be described as follows. Let $M$ be the set containing all resources and $\mathrm{M}_{0}$ the set of resources that have already been scheduled.

step $0 \mathrm{M}_{0}=\{\}$

step 1 Identify the bottleneck resource $\mathrm{m}$ among resources $\mathrm{MM}_{0}$, and calculate its optimal sequence, given the partial schedule on resources $M_{0}$. Set $M_{0}=M_{0}+\{m\}$.

step 2 Reoptimize successively the sequence of each resource $\mathrm{k} \in \mathrm{M}_{0}$, given the partial schedule on resources $M_{0} \backslash\{k\}$. If $M_{0}=M$ then stop, else go to step 1 .

The shifting bottleneck procedure schedules resources consecutively, one at a time. At each iteration, two decisions have to be taken in step 1, namely, we have to decide which resource is the bottleneck and we have to determine the sequence on that resource. A particular one-machine problem is used for both decisions. $M_{0}$ is the set of resources that are already scheduled. Each resource $j \in M_{0}$ has a selection $E_{j}$. Let $P\left(k, M_{0}\right)$ be the minimum makespan problem obtained from DG by first replacing all disjunctive arcs of resources $M_{0} \backslash\{k\}$ by the corresponding selections $E_{k}{ }^{\prime}$ and second by discarding all disjunctive arcs of resources $j \in M M_{0}$ and $j \neq k$. The bottleneck $m$ is the resource with the largest makespan for problem $\mathrm{P}\left(\mathrm{k}, \mathrm{M}_{0}\right)$, i.e. the resource $\mathrm{m}$ for which $\mathrm{P}\left(\mathrm{m}, \mathrm{M}_{0}\right)=\max _{\mathrm{k} \in \mathrm{MM} 0} \mathrm{P}\left(\mathrm{k}, \mathrm{M}_{0}\right)$. The sequence on resource $m$ is the sequence found by $P\left(m, M_{0}\right)$. The one-machine problem, $P\left(k, M_{0}\right)$, can be formulated as follows: 


$$
\begin{array}{llll}
\mathrm{P}\left(\mathrm{k}, \mathrm{M}_{0}\right) & \min & \mathrm{C} & \\
& & \\
& \mathrm{S} . \mathrm{T} . & \mathrm{C} \geq \mathrm{t}_{\mathrm{i}}+\mathrm{p}_{\mathrm{i}}+\mathrm{q}_{\mathrm{i}} & \forall \mathrm{i} \in \mathrm{N}_{\mathrm{k}} \\
& \mathrm{t}_{\mathrm{i}} \geq \mathrm{t}_{\mathrm{j}}+\mathrm{p}_{\mathrm{j}} \vee \mathrm{t}_{\mathrm{j}} \geq \mathrm{t}_{\mathrm{i}}+\mathrm{p}_{\mathrm{i}} & \forall(\mathrm{i}, \mathrm{j}) \in \mathrm{E}_{\mathrm{k}} \\
& \mathrm{t}_{\mathrm{i}} \geq \mathrm{r}_{\mathrm{i}} & \forall \mathrm{i} \in \mathrm{N}_{\mathrm{k}}
\end{array}
$$

with $\mathrm{C}$ the makespan

$\mathrm{N}_{\mathrm{k}}$ the set of all operations requiring resource $\mathrm{k}$

$t_{i} \quad$ the starting time of operation $i$

$r_{i}$ the head of operation $i$, or the length of the longest path from $\{b\}$ to operation $i$, given the partial schedule determined by resources $\mathbf{M}_{0}$

$q_{i}$ the tail of operation $i$, or the length of the longest path from operation $i$ to \{e\}, given the partial schedule determined by resources $M_{0}$

For the standard job shop problem, heads (tails), $r_{i}\left(q_{i j}\right)$, can be calculated recursively in $O(n)$ time, since each operation can have at most two predecessors (successors). Heads and tails are recalculated frequently, since they change each time that the partial schedule changes.

$\mathrm{P}\left(\mathrm{k}, \mathrm{M}_{0}\right)$ can be solved by a branch and bound algorithm of Carlier (1982) or Grabowski et al. (1986). Other references dealing with this problem are McMahon \& Florian (1975) and Potts (1980). We implemented the branch and bound algorithm of Carlier (1982). Although $\mathrm{P}\left(\mathrm{k}, \mathrm{M}_{0}\right)$ is NP-hard, fairly large problems can be solved within reasonable time.

Step 2 is a local re-optimization procedure. Let $r(1), \ldots, r(p)$ be an ordering of $M_{0}\left(p=\left|M_{0}\right|\right)$. $\mathrm{IM}_{0} \mid$ stands for the cardinality of set $\mathrm{M}_{0}$. More specifically, the local re-optimization cycle can be summarized as follows :

for $i=1, \ldots, p$ solve problem $P\left(r(i), M_{0} \backslash\{r(i)\}\right)$ and substitute the selection of $r(i)$ by the solution obtained.

At each iteration, the re-optimization cycle tries to improve the sequence of resource $r(\mathrm{i})$, given the sequences on the other resources in $M_{0}$. Each evaluation of $P\left(r(i), M_{0} \backslash\{r(i)\}\right)$ requires a full recalculation of heads and tails. The cycle is repeated a number of times (e.g. 3 times when $\left|\mathrm{M}_{0}\right|<|\mathrm{M}|$ and until no further improvement can be obtained when $\left.\left|\mathrm{M}_{0}\right|=|\mathrm{M}|\right)$.

Improvements of the SBP are proposed by Dauzère-Péres \& Lasserre (1993) and Balas et al. (1992). The one-machine problem treats all operations as independent, while it is possible that there exists in the partial solution a path between two operations to be sequenced. Such paths result in delayed precedence constraints. Including these constraints might provide higher quality solutions. Dauzère-Péres \& Lasserre (1993) have developed the modified shifting bottleneck procedure which takes into account delayed precedence constraints. Balas et al. (1992) propose a branch and bound algorithm for solving the one-machine problem with delayed precedence constraints. 


\subsection{A SBP Procedure for the EDG}

In this section, we propose an adapted shifting bottleneck procedure for finding solutions to the problem represented by the EDG. The main logic of the original SBP is maintained, except for two important modifications : the solutions for the one-machine problem and the head and tail calculations. For the time being, we do not consider sequence dependent setup times (i.e. all S labels in the EDG are zero) and we do not include delayed precedence constraints.

\subsubsection{Parallel Machine Problems}

At each step of the procedure, we will schedule one resource. However, since we allow parallel machining, it is required to solve parallel machine problems instead of one-machine problems. More specifically, instead of solving problems of the type $P\left(k, M_{0}\right)$, we solve a parallel machine problem $\operatorname{PR}\left(k, M_{0}\right)$ whenever $R_{k}>1$.

$\operatorname{PR}\left(\mathrm{k}, \mathrm{M}_{0}\right) \quad \min \quad \mathrm{C}$

$$
\begin{aligned}
& \text { S.T. } \quad C \geq \mathrm{t}_{\mathrm{i}}+\mathrm{p}_{\mathrm{i}}+\mathrm{q}_{\mathrm{i}} \quad \forall \mathrm{i} \in \mathrm{N}_{\mathrm{k}} \\
& \mathrm{t}_{\mathrm{i}} \geq \mathrm{r}_{\mathrm{i}} \quad \forall \mathrm{i} \in \mathrm{N}_{\mathrm{k}} \\
& \mathrm{t}_{\mathrm{j}} \geq \mathrm{t}_{\mathrm{i}}+\mathrm{p}_{\mathrm{i}}+\mathrm{L}\left(\mathrm{y}_{\mathrm{ijz}}-1\right) \quad \forall(\mathrm{i}, \mathrm{j}) \in \mathrm{E}_{\mathrm{k}}, \mathrm{z}=1 . . \mathrm{R}_{\mathrm{k}} \\
& \mathrm{t}_{\mathrm{i}} \geq \mathrm{t}_{\mathrm{j}}+\mathrm{p}_{\mathrm{j}}+\mathrm{L}\left(\mathrm{y}_{\mathrm{jz}}-1\right) \quad \forall(\mathrm{i}, \mathrm{j}) \in \mathrm{E}_{\mathrm{k}}, \mathrm{z}=1 . . \mathrm{R}_{\mathrm{k}} \\
& \mathrm{y}_{\mathrm{ijz}}+\mathrm{y}_{\mathrm{jiz}} \leq 1 \quad \forall(\mathrm{i}, \mathrm{j}) \in \mathrm{E}_{\mathrm{k}}, \mathrm{z}=1 . . \mathrm{R}_{\mathrm{k}} \\
& \mathrm{x}_{\mathrm{iz}}+\mathrm{x}_{\mathrm{jz}}-1 \leq \mathrm{y}_{\mathrm{ijz}}+\mathrm{y}_{\mathrm{jz}} \quad \forall(\mathrm{i}, \mathrm{j}) \in \mathrm{E}_{\mathrm{k}}, \mathrm{z}=1 . . \mathrm{R}_{\mathrm{k}} \\
& \sum_{\mathrm{z}} \mathrm{x}_{\mathrm{iz}}=1 \\
& \mathrm{y}_{\mathrm{ijz}}, \mathrm{x}_{\mathrm{iz}} \in\{0,1\} \quad \forall(\mathrm{i}, \mathrm{j}) \in \mathrm{E}_{\mathrm{l}}, \mathrm{z}=1 . . \mathrm{R}_{\mathrm{k}}
\end{aligned}
$$

with

C the makespan

$t_{i} \quad$ the starting time of operation $i$

$r_{i}$ the head of operation $i$, or the length of the longest path in EDG from $\{b\}$ to operation $i$, given the partial schedule determined by resources $M_{0}$

$\mathrm{q}_{\mathrm{i}}$ the tail of operation $i$, or the length of the longest path in EDG from operation $i$ to $\{e\}$, given the partial schedule determined by resources $M_{0}$

$\mathrm{x}_{\mathrm{iz}}=1$ if operation $\mathrm{i}$ is assigned to the $\mathrm{z}^{\text {th }}$ unit of resource $\mathrm{k}$ $=0 \quad$ else

$\mathrm{y}_{\mathrm{ijz}}=1$ if both operations $\mathrm{i}$ and $\mathrm{j}$ are assigned to the $\mathrm{z}^{\text {th }}$ unit of resource $\mathrm{k}$ and operation $\mathrm{i}$ precedes operation $\mathrm{j}$

$=0 \quad$ else

L a sufficiently large number 
Constraints (4)-(7) force operation i either to precede or succeed operation $\mathrm{j}$, only if they are both assigned to the same unit of the resource. Constraint (8) specifies that each operation has to be assigned to one unit of the resource.

Problem PR $\left(k, M_{0}\right)$ is the parallel machine equivalent of the one-machine problem. One could apply a branch and bound algorithm to find optimal solutions to the problem. However, it is less structured than the one machine problem, and a fast branch and bound algorithm is not readily available. Therefore, we simply apply a longest tail heuristic to solve the problem, similar to the one applied to the one-machine problem (Carlier 1982). Future research will provide better algorithms for $\operatorname{PR}\left(k, M_{0}\right)$, which will result in better solutions to the EDG. The longest tail heuristic for the parallel machine problem can be described as follows.

$\mathrm{N}_{\mathrm{k}}$ is the set of all operations requiring resource $\mathrm{k}$. Let $\mathrm{U}$ be the set of scheduled operations. $t$ represents the time. Let RL be a list of eligible operations, i.e. operations which have a head smaller than $t$, but which are not yet scheduled. $t_{i}$ is the starting time of operation $i$. IP is the set of operations in process at the resource, i.e. all scheduled operations which finish later than $\mathrm{t}$.

$$
\begin{aligned}
& \text { step } 0 \quad \mathrm{U}=\{\} \\
& \mathrm{RL}=\{\} \\
& \mathrm{IP}=\{\} \\
& \mathrm{t}=0 \\
& \text { let } i^{*} \text { be an operation in RL with } q_{i^{*}}=\max i \in R L q_{i} \\
& \mathrm{IP}=\mathrm{IP}+\left\{\mathrm{i}^{*}\right\} \\
& \mathrm{U}=\mathrm{U}+\left\{\mathrm{i}^{*}\right\} \\
& R L=R L \backslash\left\{i^{*}\right\} \\
& \mathrm{t}_{\mathrm{i}^{*}}=\mathrm{t}
\end{aligned}
$$

step 3

if $\mathrm{U}=\mathrm{N}_{\mathrm{k}}$ then stop, else go to step 1

Step 0 is the initialization. In step 1 , the time is updated. $t$ is set equal to the maximum of the earliest finishing time of the operations in process and the earliest starting time of all unscheduled operations. Then, the sets IP and RL are updated, given the new $t$ value. In step 2 , ready operations are scheduled following a longest tail priority, as long as there are some units of the resource idle. The procedure ends in step 3 if all operations are scheduled.

For a parallel machine problem, not all disjunctive arcs $E_{k}$ have to be selected, but only those arcs between operations assigned to the same unit of the resource. In terms of the formulation (2)-(9), a disjunctive arc is replaced by an ordinary $\operatorname{arc}(\mathrm{i}, \mathrm{j})$ if $\mathrm{y}_{\mathrm{ijz}}=1$ for some $\mathrm{z}$. 


\subsubsection{Head and Tail Calculations}

The EDG has labels on the arcs as well as on the nodes. Let PRED $\left(\right.$ SUCC $\left._{i}\right)$ denote the conjunctive predecessors (successors) of node $i$ in the EDG. MPRED (MSUCC $_{i}$ ) is the set of disjunctive predecessors (successors) of node i, i.e. predecessors (successors) originating from a selection of disjunctive arcs in a partial solution.

The recursive head and tail calculations, including the labels on the arcs can be summarized as follows :

$$
\begin{aligned}
& r_{i}=\max \left(\max _{j \in \text { PREDi }}\left(r_{j}+p_{j}+F_{j i}\right), \max _{j \in \text { MPREDi }}\left(r_{j}+p_{j}+S_{j i}\right)\right) \\
& r_{b}=0 \\
& q_{i}=\max \left(\max _{j \in S U C C i}\left(q_{j}+p_{j}+F S_{i j}\right), \max _{j \in M S U C C i}\left(q_{j}+p_{j}+S_{i j}\right)\right) \\
& q_{e}=0
\end{aligned}
$$

$\left|M P R E D_{i}\right|$ and $\left|M S U C C_{i}\right| \leq 1$, since we only need to consider the immediate disjunctive predecessor and successor of $i$.

\subsubsection{A Numerical Example}

In this section, we illustrate the SBP on the EDG example of section 2.3.7.. We describe only the bottleneck identification step of the procedure. The reoptimization step has the same logic. Suppose that one resource is already scheduled in a previous iteration, and let this resource be $M 3$, i.e. $M_{0}=\{M 3\}$. Resource $M 3$ has 2 units available. The sequence on the first unit is 7-19-16-26 and on the second unit 3-11-22. Figure 8 represents this partial solution. The disjunctive arcs of resource M3 are replaced by the corresponding selections. We have only drawn the immediate disjunctive predecessors and successors, since these are sufficient to calculate the heads and tails.

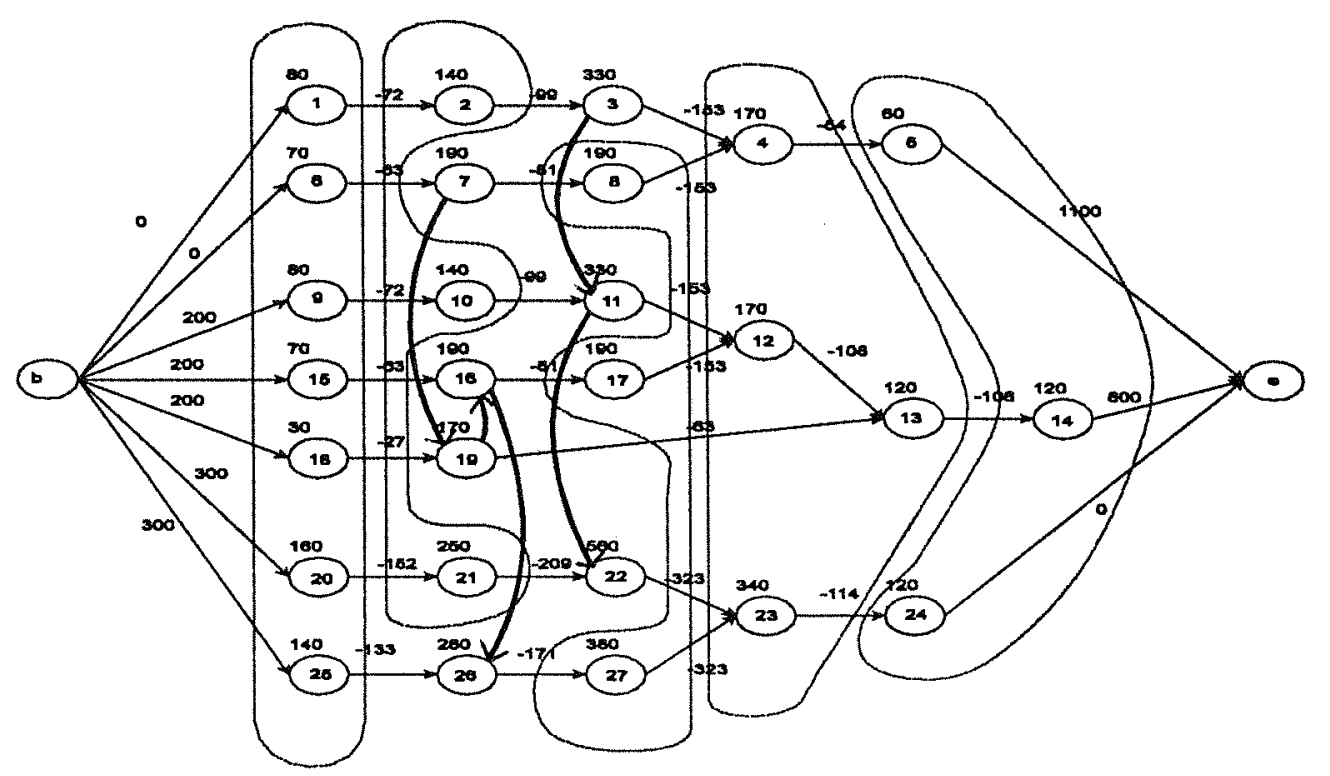

Figure $8:$ The EDG with $M_{0}=\{\mathrm{M} 3\}$ 
We will now determine the next bottleneck resource. The heads and tails are :

\begin{tabular}{|c|c|c|c|c|c|c|c|c||}
\hline $\mathrm{i}$ & $\mathrm{r}_{\mathrm{i}}$ & $\mathrm{q}_{\mathrm{i}}$ & $\mathrm{i}$ & $\mathrm{r}_{\mathrm{i}}$ & $\mathrm{q}_{\mathrm{i}}$ & $\mathrm{i}$ & $\mathrm{r}_{\mathrm{i}}$ & $\mathrm{q}_{\mathrm{i}}$ \\
\hline \hline 1 & 0 & 1470 & 10 & 208 & 1072 & 19 & 203 & 1140 \\
\hline 2 & 8 & 1402 & 11 & 379 & 841 & 20 & 300 & 472 \\
\hline 3 & 49 & 1171 & 12 & 556 & 824 & 21 & 308 & 347 \\
\hline 4 & 226 & 1106 & 13 & 618 & 812 & 22 & 709 & 23 \\
\hline 5 & 342 & 1100 & 14 & 630 & 800 & 23 & 946 & 6 \\
\hline 6 & 0 & 1550 & 15 & 200 & 1077 & 24 & 1172 & 0 \\
\hline 7 & 7 & 1310 & 16 & 373 & 950 & 25 & 300 & 379 \\
\hline 8 & 116 & 1123 & 17 & 482 & 841 & 26 & 563 & 232 \\
\hline 9 & 200 & 1140 & 18 & 200 & 1283 & 27 & 672 & 23 \\
\hline
\end{tabular}

Table IV : The Heads and Tails

The next bottleneck is the machine with the highest makespan on its one-machine or parallel machine problem. Consider for example resource M1. There are 2 units available of this resource and operations $1,6,9,15,18,20$ and 25 have to be scheduled. Applying the longest tail heuristic gives the sequence 1-18-15-20 on the first unit and 6-9-25 on the second resource, with starting times $t_{1}=0, t_{6}=0, t_{9}=200, t_{15}=230, t_{18}=200, t_{20}=300, t_{25}=300$. The corresponding makespan is 1550 . The makespan of all other unscheduled resources is given in table $\mathrm{V}$.

\begin{tabular}{|c|c|}
\hline Resource & Cmax \\
\hline \hline M1 & 1550 \\
\hline M2 & 1550 \\
\hline M4 & 1513 \\
\hline M5 & 1658 \\
\hline M6 & 1550 \\
\hline
\end{tabular}

Table V:The Makespans of the Unscheduled Resources

Thus, the next bottleneck is M5. The sequence on this resource is 4-12-13-23. 


\section{Computational Results}

In this section, we compare the performance of the shifting bottleneck procedure with priority dispatching rules on a set of large problems.

\subsection{Generation of the Test Problems}

We have generated randomly 4 sets of 21 problems. The problems are numbered from 1 to 84 and have the dimensions as shown in table VI. The first set consists of problems 1-21, set 2 contains problems 22-42, set 3 deals with problems $43-63$ and the set 4 includes problems 64-84.

Problems
\begin{tabular}{|r|r|r|r|r|}
\hline \multicolumn{7}{|c|}{} & Products & Resources & Orders \\
\hline Set1 & Set2 & Set3 & Set4 & \\
\hline
\end{tabular}
\begin{tabular}{|r|r|r|r|r|r|r|}
\hline 1 & 22 & 43 & 64 & 20 & 40 & 50 \\
\hline 2 & 23 & 44 & 65 & 20 & 40 & 50 \\
\hline 3 & 24 & 45 & 66 & 20 & 40 & 50 \\
\hline 4 & 25 & 46 & 67 & 25 & 50 & 50 \\
\hline 5 & 26 & 47 & 68 & 25 & 50 & 50 \\
\hline 6 & 27 & 48 & 69 & 25 & 50 & 50 \\
\hline 7 & 28 & 49 & 70 & 30 & 60 & 100 \\
\hline 8 & 29 & 50 & 71 & 30 & 60 & 100 \\
\hline 9 & 30 & 51 & 72 & 30 & 60 & 100 \\
\hline 10 & 31 & 52 & 73 & 35 & 70 & 100 \\
\hline 11 & 32 & 53 & 74 & 35 & 70 & 100 \\
\hline 12 & 33 & 54 & 75 & 35 & 70 & 100 \\
\hline 13 & 34 & 55 & 76 & 40 & 80 & 200 \\
\hline 14 & 35 & 56 & 77 & 40 & 80 & 200 \\
\hline 15 & 36 & 57 & 78 & 40 & 80 & 200 \\
\hline 16 & 37 & 58 & 79 & 45 & 90 & 200 \\
\hline 17 & 38 & 59 & 80 & 45 & 90 & 200 \\
\hline 18 & 39 & 60 & 81 & 45 & 90 & 200 \\
\hline 19 & 40 & 61 & 82 & 50 & 100 & 300 \\
\hline 20 & 41 & 62 & 83 & 50 & 100 & 300 \\
\hline 21 & 42 & 63 & 84 & 50 & 100 & 300 \\
\hline
\end{tabular}

\section{Table VI : The Test Problems}

For all problems, the release dates of the orders are set equal to zero. Problem sets 1-21 and 43-63 have no due dates, so the objective is to minimize Cmax. Problems 22-42 and 64-84 correspond to the sets 1-21 and 43-63, but have due dates and are solved with respect to the min Lmax objective (e.g. problem 22 is the same as problem 1, but with due dates). The due dates setting is based on the solutions obtained on the original problems. For each order, we draw a uniformly distributed random number between the minimal time required for completing the order (i.e. the longest path in that part of the disjunctive graph originating from the order) and the makespan obtained from the min Cmax problem. By doing so, we obtain tight due dates.

Problems 1-21 and 22-42 have no overlapping operations and the setup times are set equal to zero. Problems 43-63 and 64-84 on the other hand do allow for overlapping operations and non-zero setup times. 
To give the reader an idea of the dimensions involved, take e.g. test problem 15 with 40 products, 80 resources and 200 orders. The random generation of this problem resulted in a disjunctive graph with 1629 nodes. Details about the generation of the test problems can be obtained from the authors.

\subsection{The Solution Procedures Tested}

We tested the SBP against a collection of priority dispatching rules (Panwalker \& Iskander 1977, Blackstone et al. 1982). We implemented a simple schedule generation procedure (SGP). The procedure starts at the beginning of the time horizon and increments the time until all operations are scheduled. When a resource conflict is encountered, some operations are delayed to solve the conflict. The delay decisions are based on a primary priority rule and a tie breaker priority rule. We applied 19 rules: SPT (shortest processing time), LPT (longest processing time), FCFS (first come first served), MOR (most operations remaining), LOR (least operations remaining), MTO (most total operations), LTO (least total operations), MWR (most work remaining), LWR (least work remaining), MTW (most total work), LTW (least total work), TOP (total operations over imminent processing time), WRP (work remaining over imminent processing time), EDD (earliest due date), SLACK (slack), SLKOR (slack over operations remaining), SLKWR (slack over work remaining), SLKRAT (slackratio, slack over due date), RAN (random). We solved all test problem two times for each priority rule, once with SPT as tie breaker and once with LPT as tie breaker, totalling 38 SGP runs for each test problem. We recorded the best outcome over all runs as well as the average performance over all runs and compared these figures with the outcome of the SBP.

We implemented a simple version of the shifting bottleneck procedure (Adams et al. 1988), without delayed precedence constraints (Dauzère-Péres \& Lasserre 1993, Balas et al. 1992). We used a longest tail heuristic to solve the parallel machine problems. For the one-machine problems, we applied the branch and bound procedure of Carlier (1982). Since the instances are rather large, we limited the number of nodes in the branch and bound tree to 200 (i.e. we backtrack when there are 200 nodes in the tree).

\subsection{The Results}

Table VII shows the results for the test problems 1-21. Since these problems have no due dates, the objective is to minimize the makespan of the schedule. Column (1) is the makespan found by the SBP. Column (2) is the average makespan of the 38 SGP runs (per problem). Column (3) displays the improvement of the SBP relative to the average SGP makespan. On average, the SBP found a makespan that was $18.12 \%$ shorter than the average SGP makespan. It is interesting to note that SGP found the same solution on only 4 instances and in only 2 out of 21 cases, the SBP solution was worse (of course compared to the best solution out of the 38 SGP runs per problem).

The results for problems $22-42$ are shown on table VIII. Remember that these problems are the same as 1-21, but now with due dates. The objective was to minimize the maximum lateness. Since the due dates are rather tight, all solutions (except the SBP solution to problem 33) have late orders. Compared to the average SGP solution, the SBP was $80.57 \%$ 
better, which is not surprising since we included a number of priority rules which do not contain any due date information.

\begin{tabular}{|c|c|c|c|}
\hline \multicolumn{4}{|c|}{ et 1 objective min Cmax } \\
\hline & (1) & (2) & (3) \\
\hline Problem & SBP & Avg SGP & {$[(2)-(1)] /(2) \%$} \\
\hline 1 & 12847 & 14714 & 12.69 \\
\hline 2 & 12125 & 15031 & 19.33 \\
\hline 3 & 19898 & 23929 & 16.85 \\
\hline 4 & 9264 & 10951 & 15.40 \\
\hline 5 & 8376 & 13391 & 37.45 \\
\hline 6 & 26987 & 34121 & 20.91 \\
\hline 7 & 13054 & 15385 & 15.15 \\
\hline 8 & 13780 & 16176 & 14.81 \\
\hline 9 & 34040 & 41929 & 18.82 \\
\hline 10 & 29510 & 33320 & 11.43 \\
\hline 11 & 12175 & 15941 & 23.62 \\
\hline 12 & 21530 & 29115 & 26.05 \\
\hline 13 & 92469 & 106801 & 13.42 \\
\hline 14 & 30976 & 32697 & 5.26 \\
\hline 15 & 41464 & 45846 & 9.56 \\
\hline 16 & 60478 & 64415 & 6.11 \\
\hline 17 & 49226 & 54474 & 9.63 \\
\hline 18 & 14192 & 17532 & 19.05 \\
\hline 19 & 39256 & 67026 & 41.43 \\
\hline 20 & 43356 & 51379 & 15.62 \\
\hline 21 & 25075 & 34810 & 27.97 \\
\hline & & Average & 18.12 \\
\hline
\end{tabular}

Table VII : The Results for Problems 1-21

Set 2 : objective $\min L \max$

\begin{tabular}{|c|c|c|c|}
\cline { 2 - 4 } \multicolumn{1}{c|}{} & $(1)$ & $(2)$ & $(3)$ \\
\hline Problem & SBP & Avg SGP & {$[(2)-(1)] /(2) \%$} \\
\hline
\end{tabular}

\begin{tabular}{|r|r|r|r|}
\hline 22 & 1670 & 8743 & 80.90 \\
\hline 23 & 3415 & 6261 & 45.46 \\
\hline 24 & 1959 & 10063 & 80.53 \\
\hline 25 & 2057 & 5402 & 61.92 \\
\hline 26 & 1715 & 6044 & 71.62 \\
\hline 27 & 4752 & 18956 & 74.93 \\
\hline 28 & 2483 & 8732 & 71.56 \\
\hline 29 & 1612 & 7664 & 78.97 \\
\hline 30 & 4140 & 24440 & 83.06 \\
\hline 31 & 4116 & 20958 & 80.36 \\
\hline 32 & 1121 & 6355 & 82.36 \\
\hline 33 & -406 & 18475 & 102.20 \\
\hline 34 & 9621 & 71147 & 86.48 \\
\hline 35 & 2700 & 19187 & 85.93 \\
\hline 36 & 2342 & 28412 & 91.76 \\
\hline 37 & 4514 & 41342 & 89.08 \\
\hline 38 & 7681 & 32722 & 76.53 \\
\hline 39 & 2368 & 10037 & 76.41 \\
\hline 40 & 533 & 47315 & 98.87 \\
\hline 41 & 3985 & 27818 & 85.67 \\
\hline 42 & 2596 & 20713 & 87.47 \\
\hline & & Average & \\
\hline & & & 80.57 \\
\hline
\end{tabular}

Table VIII : The Results for Problems $22-42$ 
Tables IX and X are the outcomes for the problem sets 3 and 4 . The results on these sets confirm our findings on the sets 1 and 2. For the set 3, with an objective of min Cmax, the SBP finds on average solutions that where $7.75 \%$ better than the SGP runs. On problem set 4 , the SBP results in Lmax values that are on average $87.02 \%$ better. Totalled over sets 3 and 4 , the SBP finds a slightly worse solution than the best SGP run in only 6 out of the 42 cases.

\begin{tabular}{|c|c|c|c|}
\hline & (1) & (2) & (3) \\
\hline Problem & $\overline{S B P}$ & Avg SGP & {$[(2)-(1)] /(2) \%$} \\
\hline 43 & 7130 & 8066 & 11.60 \\
\hline 44 & 9253 & 9961 & 7.11 \\
\hline 45 & 11664 & 12065 & 3.32 \\
\hline 46 & 12213 & 12590 & 2.99 \\
\hline 47 & 19750 & 20472 & 3.53 \\
\hline 48 & 9088 & 9888 & 8.09 \\
\hline 49 & 21832 & 22720 & 3.91 \\
\hline 50 & 16940 & 18123 & 6.53 \\
\hline 51 & 16232 & 16762 & 3.16 \\
\hline 52 & 33807 & 34522 & 2.07 \\
\hline 53 & 21270 & 26170 & 18.72 \\
\hline 54 & 14519 & 14607 & 0.60 \\
\hline 55 & 41058 & 42827 & 4.13 \\
\hline 56 & 18555 & 20379 & 8.95 \\
\hline 57 & 34491 & 36759 & 6.17 \\
\hline 58 & 16113 & 16873 & 4.50 \\
\hline 59 & 91931 & 96426 & 4.66 \\
\hline 60 & 48077 & 48981 & 1.85 \\
\hline 61 & 19585 & 33922 & 42.26 \\
\hline 62 & 90627 & 92779 & 2.32 \\
\hline 63 & 59207 & 70650 & 16.20 \\
\hline & & & 7.75 \\
\hline
\end{tabular}

Table IX : The Results for Problems 43-63

The computational effort required by the SBP was considerably higher then the SGP. We implemented both heuristics in C, compiled on a $48633 \mathrm{Mhz} P C$ under the Microsoft WindowsNT operating system. On average over the 84 test problems, the SBP required 191 seconds per run, compared to approximately 5 seconds per SGP run. However, the solution obtained by the SBP outperforms the solutions of the SGP runs, and the total time for these 38 runs was $18 * 4.82=183$ seconds, which comes in the neighborhood of the SBP time. The average SBP time required for the largest problems (50 products, 100 resources, 300 orders) was around 7 minutes for problem sizes of approximately 2500 nodes in the EDG. Given the size of the test problems and the number of resources involved, we can conclude that the computational effort of the SBP is small and consequently very promising for real-life applications. 
Set 4 : objective $\min$ Lmax

\begin{tabular}{|r|r|r|r|}
\hline \multicolumn{1}{c|}{} & \multicolumn{1}{c|}{$(1)$} & \multicolumn{1}{c|}{$(2)$} & \multicolumn{1}{c|}{$(3)$} \\
\hline Problem & SBP & Avg SGP & [(2)-(1)]/(2) \% \\
\hline 64 & -130 & 3317 & 103.92 \\
\hline 65 & 1654 & 5101 & 67.57 \\
\hline 66 & 1378 & 4678 & 70.54 \\
\hline 67 & -410 & 6436 & 106.37 \\
\hline 68 & -482 & 10446 & 104.61 \\
\hline 69 & 1397 & 4806 & 70.93 \\
\hline 70 & -382 & 11592 & 103.30 \\
\hline 71 & 1511 & 9468 & 84.04 \\
\hline 72 & 1571 & 9159 & 82.85 \\
\hline 73 & 1048 & 19951 & 94.75 \\
\hline 74 & 647 & 15433 & 95.81 \\
\hline 75 & 1536 & 6722 & 77.15 \\
\hline 76 & 3158 & 14284 & 77.89 \\
\hline 77 & 2895 & 13271 & 78.19 \\
\hline 78 & 2320 & 19508 & 88.11 \\
\hline 79 & 2571 & 9857 & 73.92 \\
\hline 80 & 4159 & 58101 & 92.84 \\
\hline 81 & 1731 & 26679 & 93.51 \\
\hline 82 & 1509 & 23945 & 93.70 \\
\hline 83 & 3377 & 61364 & 94.50 \\
\hline 84 & 11352 & 41822 & 72.86 \\
\hline & & & \\
\hline & & & \\
\hline & & & \\
\hline & & & \\
\hline
\end{tabular}

Table X : The Results for Problems 64-84

\section{Conclusion}

The objective of this paper is to modify the Shifting Bottleneck Procedure so that problem characteristics such as setup times, overlapping, beginning inventory, assembly and split structures, internal transportation delays and parallel machining can be handled. The above mentioned characteristics of real-life applications can be easily modeled by means of a disjunctive graph representation. This is mainly done through the introduction of finish-start precedence relationships, resulting in a label for the arcs in the disjunctive graph.

The SBP has to be modified in two ways. First, an adaption is required for the head and tail computations and second, the one-machine subproblems have to be substituted by an algorithm to solve the parallel machine problem. The computational experience, based on a set of 84 test problems shows that this extended SBP outperformed schedule generation procedures (38 priority dispatching rules per problem) for almost all problems tested. Moreover, the computation time required is such that even for large real-life cases the SBP algorithm will not constitute a major obstacle. The performance of the extended SBP can further be improved by refining the longest tail heuristic used to solve the parallel machine problems. A branch and bound algorithm for this problem would be suitable. Moreover, a better treatment of the delayed precedence constraints in the disjunctive graph (Dauzère-Péres \& Lasserre 1993, Balas et al. 1992) may further enhance the solution procedure. 
The main conclusion however is that the extended SBP and the underlying disjunctive graph representation offer a very powerful tool to model and to solve real-life general job shop scheduling problems.

\section{References}

Adams J., Balas E., Zawack D., The Shifting Bottleneck Procedure for Job-Shop Scheduling, 1988, Management Science, Vol. 34, No. 3, pp. 391-401

Applegate D., Cook W., A Computational Study of the Job-Shop Scheduling Problem, 1991, ORSA Journal on Computing, Vol. 3, No. 2, pp. 149-156

Balas E., Lenstra J.K., Vazacopoulos A., One Machine Scheduling with Delayed Precedence Constraints, 1992, Management Science research Report \#MSRR-589, Carnegie Mellon University

Balas E., On the Facial Structure of Scheduling Polyhedra, 1985, Mathematical Programming Study 24, pp. 179. 218

Barker J.R., McMahon G.B., Scheduling the General Job-Shop, 1985, Management Science, Vol. 35, No. 5

Blackstone J.H., Phillips D.T., Hogg G.L., A State-of-the-art Survey of Dispatching Rules for Manufacturing Job Shop Operations, 1982, International Journal of Production Research, Vol. 20, No. 1, pp.27-45

Brucker P., Jurisch B., Krämer A., The Job-Shop Problem and Immediate Selection, 1992, Osnabrücker Schriften zur Mathematik, P 145

Brucker P., Jurisch B., Sievers B., A Fast Branch-and-Bound Algorithm for the Job-Shop Scheduling Problem, 1991, Osnabrücker Schriften zur Mathematik, P 136

Carlier J., The One-Machine Sequencing Problem, 1982, European Journal of Operational Research, Vol. 11, pp. $42-47$

Carlier J., Pinson E., An Algorithm for Solving the Job-Shop Problem, 1989, Management Science, Vol. 35, No. 2, pp. 164-176

Dauzère-Péres S., Lasserre J.B., A Modified Shifting Bottleneck Procedure for Job-Shop Scheduling, 1993, International Journal of Production Research, Vol. 31, No. 4, pp. 923-932

Grabowski J., Nowicki E., Zdrzalka S., A Block Approach for Single-Machine Scheduling with Release Dates and Due-Dates, 1986, European Journal of Operational Research, Vol. 26, pp. 278-285

Lageweg B.J., Lenstra J.K., Rinnooy Kan A.H.G., Job-Shop Scheduling by Implicit Enumeration, 1977, Management Science, Vol. 24, pp. $441-450$

McMahon G., Florian M., On Scheduling with ready Times and Due Dates to Minimize Maximum Lateness, 1975, Operations Research, Vol. 23, pp. 475-482

Panwalker S. S., Iskander W., A Survey of Scheduling Rules, 1977, Operations Research, Vol. 25, pp.45-61

Potts C.N., Analysis of a Heuristic for One Machine Sequencing with Release Dates and Due-Dates to Minimize Maximum Lateness, 1980, Operations Research, Vol. 23, pp. 1436-1441

Van Laarhoven P.J.M., Aarts E.H.L., Lenstra J.K., Job-Shop Scheduling by Simulated Annealing, 1992, Operations Research, Vol. 40, No. 1, pp. 113-125 